\title{
Inhalt
}

\section{Teilband I}

Vorwort - XIII

Schriftenverzeichnis - XVII

Maha Akeel

Two Demotic Ostraca from the Roman Period - 1

Hartwig Altenmüller

Zur apotropäischen Geste der Hirten in der Furt - Die Reden und Rufe der Hirten im Grab des Wesirs Hesi in Saqqara — 11

Susanne Beck \& Kathrin Gabler

Die Überlieferung von Text und Befund in Deir el-Medine am Beispiel des Wächters Khawy/H3wj (ii) 29

Elke Blumenthal

Frömmigkeit und Magie in pharaonischer Zeit $\longrightarrow 79$

Isa Böhme

Botschafter der kosmischen Ordnung - Thot, Geb, Horus und und Tb 161 auf den privaten Sarkophagen des Neuen Reiches - 103

Nadja Braun

Zehn Jahre Narrenfreiheit - Kinder im alten Ägypten — 133

Alexander Brawanski

Die Schreiber des Papyrus Edwin Smith. Eine statistische Analyse der Paläographie des chirurgischen Abschnittes — 151

Marc Brose

Periphrastische Konstruktionen mit $j$ ri $i$,machen; tun“ vor der Amarnazeit - 175

Silke Caßor-Pfeiffer \& Stefan Pfeiffer

Pharaonin Berenike II.: Bemerkungen zur ägyptischen Titulatur einer

frühptolemäischen Königin — 199 
Christina Di Cerbo \& Richard Jasnow

Building Dipinti at Medinet Habu (With an Appendix on Remains of Color

Decoration in the Palace) -239

Peter Dils \& Gunnar Sperveslage

Forschungsgeschichtliche Beobachtungen zur Erschließung der hieroglyphischen Lautwerte am Beispiel des Braunen Sichlers (G28, gm) - 261

Koenraad Donker van Heel

Choachytes lower middle class? No way José! —— 297

Andreas Dorn, Matthias Müller \& Mohammed Sherif Ali

Unbekannte und unerkannte literarische Ostraka des Neuen Reiches - 309

John Gee

Literary Titles from the Greco-Roman Period - 329

Maren Goecke-Bauer

Amunmes - EIN Deir-el-Medine-Schreiber? - 353

Martina Grünhagen

„Im Banne einer Frau sein“ als Grund unrechten Handelns - Der Fall des „Heißen“ in oBorchardt $1-375$

Jana Helmbold-Doyé

Tür-Konsolen in Architektur und bildlichen Darstellungen griechisch-römischer Zeit -385

Anja Hilbig \& Carina Kühne-Wespi

Das Grab des Schuroy (TT 13) in einem anderen Licht: Zu neuen archäologischen Ergebnissen und philologischen Betrachtungen -433

Friedhelm Hoffmann

Pa-neb-taui in Hildesheim? -453

Karl Jansen-Winkeln

Ein Wezir als Priester -461

Manfred Krebernik

Asar(i) und Osiris - 483 
Myriam Krutzsch

Erkenntnisse und Fragen zum Schriftträger Papyrus - 503

Sabine Kubisch

Visualisierte Verdienste. Zum Anbringungsort biographischer Inschriften der Ramessidenzeit $\longrightarrow \mathbf{5 1 5}$

Robert Kuhn

(Wieder-)Entdeckung. Eine ritzverzierte Schminkpalette aus Naqada aus dem Ägyptischen Museum und Papyrussammlung Berlin -531

Eva Lange-Athinodorou

Der „Tempel des Hermes“ und die Pfeile der Bastet. Zur Rekonstruktion der Kultlandschaft von Bubastis -549

Nikolaos Lazaridis

Carving the Desert: Ancient Travellers' Epigraphic Uses of Kharga Oasis Rock Surfaces -587

\section{Giuseppina Lenzo}

La déesse Hepetet(-Hor) dans les papyrus, sarcophages et tombes des $21^{\text {ème }}$ et $22^{\text {ème }}$ dynasties -601

Alexandra von Lieven

$s m . t$ - Schlafanzug für unverheiratete Männer, Hängematte oder Zelt?

Ein problematisches Lexem in der Biographie des Ahmose, Sohn der Ibana — 621

\section{Teilband II}

Ulrich Luft

Die Sozialstruktur der unteren Bevölkerungsschicht im Mittleren Reich — 631

\section{Benoît Lurson}

Le « roi-statue ». Une image d’idéologie royale au temple de Séthi ler à

Abydos -675

\section{Bernard Mathieu}

Attaquer ou ne pas attaquer? Le pharaon Kamosis au cœur d'un conflit idéologique (avec une nouvelle traduction de la « Geste de Kamosis ») - 703 
Pierre Meyrat

Un papyrus pas piqué des vers. À propos du roi Néferkarê évoqué dans le Traité d'ophiologie — 719

Jan Moje

Bemerkungen zur Entwicklungsgeschichte der demotischen Epigraphik im kulturgeschichtlichen Kontext des späten Ägypten — 733

Ludwig D. Morenz

Motivator, Vor-Bild und Trigger des Aleph. Zur kulturhistorischen Ursprungsgeschichte des Alphabets auf dem Hochplateau von Serabit el-Chadim $\longrightarrow 757$

Renate Müller-Wollermann

$h p-$ „Richtschnur“

Franziska Naether

Heliopolis in Egyptian Literary Texts: Sacred Architecture and Cult Activities - 773

Hana Navrátilová

Between patronage and an Egyptological invisible college - The early career of Jaroslav Černý 799

Boyo Ockinga

A son welcomes his father to the netherworld: an unusual funerary text from TT $233-815$

Tanja Pommerening

„Was verbirgt sich hinter der Quantenangabe ,1` in den Rezepten für ein Maß?" 831

Lutz Popko

Keine Parallele zu pHarris I. Ein Vorbericht zu oDeM 1466 verso 849

Luigi Prada

The Funerary Stela of Nephersouchis II, an Additional Member of the Family of the High Priests of Memphis (Aegyptiaca Wilkinsoniana I) -869

Joachim F. Quack

Ein Bericht über eine erfolgreiche Mission. Papyrus Inv. Sorbonne 2239abc + $2242 a-915$ 
Susanne Radestock

Übergang oder Grenze? Eine Diskussion zu den Begrifflichkeiten Hierarchie und Dichotomie bezüglich der ägyptischen Medizin — 931

Jana Raffel

„Egal was, ich war’s nicht!“ Zur Nicht-Identifikationsformel in Zusammenhang mit der Götterbedrohung - 947

Dietrich Raue

Figurinen aus Aniba im Ägyptischen Museum -Georg Steindorff- der Universität Leipzig - 965

Ilona Regulski

Timeless offerings - BM EA 1172 re-contextualised - 983

Tonio S. Richter

Ein Bittbrief und eine Lohnkalkulation auf einem koptischen Papyrus im Besitz von Ralf und Susanne Lorych — 999

Micah T. Ross

Philology and Science at Dendera - 1011

Frédéric Rouffet

Recherches sur la déesse Tabitchet — 1021

Kim Ryholt

A Self-Dedication addressed to Anubis in Berlin and Leipzig. P. Berlin P. $23742+$

P. Lips. inv. $1364-1041$

Karl-J. Seyfried

Bemerkungen zu Besitz und Anteil an Grabanlagen durch Frauen im späten Alten

Reich 1047

Martin A. Stadler

Die Götterwelt von Dimê. Papyrus Berlin P. 30006 rt. — 1061

Katharina Stegbauer

„Per Anhalter durchs Mittelmeer“ — 1091

Michael P. Streck

Grüne Tempeltürme und die Hängenden Gärten von Babylon — 1113 
Christoffer Theis

Die siebenköpfige Schlange im Vorderen Orient —-1123

Susanne Töpfer

Magika Hieratika in Kopenhagen - Sprüche zum Schutz des Leibes auf pCarlsberg $906-1137$

Ursula Verhoeven

Georg Möller (1876-1921): Spuren und Wahrnehmungen — 1159

Steve Vinson

Mastaba or Perron? On ns3.t / NHce in the "First Tale of Setne Khaemwas" and Other Demotic and Coptic Texts -1177

Günter Vittmann

Ein frühdemotisches Schultäfelchen (Louvre E 9846) — 1191

Susanne Voss

Weiße Pharaonen. Bemerkungen zu Menschenbild, Zeitgeist und Terminologie in ägyptologischen Schriften des 19. und 20. Jahrhunderts - 1211

Jean Winand

Did you say synonyms? The case of $p h$ and $s p r$ in Late Egyptian — 1235

Naoko Wolze

Rekonstruktion der Inschriften auf der Kanalstele Darius' I. aus Tell elMaskhuta -1275

Waldemar Wolze

Das steinerne Schreibpalettenfragment Inv.-Nr. 1935,200,154 des Minmose im Museum August Kestner, Hannover 1321 